\title{
Study on the characteristic curve of laser-induced delayed luminescence in leaves of Scindapsus
}

\author{
Meili Jiang ${ }^{1}$ Chenhao $\mathrm{Li}^{1} \cdot$ B. Qing Tang ${ }^{1,2} \cdot$ Peng Gao $^{1} \cdot$ Jing Wang $^{1} \cdot$ \\ Yongdong Yang ${ }^{1} \cdot$ Xiaochun Zhang ${ }^{1} \cdot$ Yang Liu $^{1} \mathbb{C}$
}

Received: 15 March 2021 / Accepted: 13 July 2021

(C) The Author(s) 2021

\begin{abstract}
At room temperature, we studied the delayed luminescence characteristic curves of the leaves of Scindapsus in darkroom and continuously irradiate at different wavelengths. The results show that the decay of stimulated emission photons induced by pulsed laser has a typical hyperbolic trend with the delay time, and the goodness of fitted curve can reach $99.9 \%$. The initial photon intensity $I(0)$ and decay time $\tau$ are closely related to the environment and physiological activities of plants. In the dark room, it was found that the initial intensity and decay time of the delayed luminescence curve for picked leaves decreased, while the potted leaves remained almost unchanged. The characteristic curve of laser-induced delayed luminescence accurately reflected the process and degree of plant wilting. For continuously irradiated by the laser wavelength of $520 \mathrm{~nm}$ and $400 \mathrm{~nm}$, respectively, the change in curve parameters reflects the selective absorption of different light wavelengths. Compare the condition with having light and without, the metabolites of photosynthesis and respiration are different in plants, which leads to greatly changes in decay time. The characteristic curve of laser-induced delayed luminescence can be used as an important parameter to measure the physiological state of plants.
\end{abstract}

Xiaochun Zhang

zhangxcb@enn.cn

$\triangle$ Yang Liu

691652136@qq.com

1 ENNOVA Institute of Life Science and Technology, ENN Group, Langfang 065001, China

2 School of Physics, Sichuan University, Chengdu 610065, China

\section{Introduction}

Biological delayed luminescence (DL) was first observed in plant by Sstrehler and Arnold, who involved the detection of light-induced Adenosine triphosphate (ATP) formation in algae samples [1]. DL was defined as the luminescence of photosynthetic materials after they were irradiated by the excitation light [2]. The DL was successively observed in other biological samples, such as leaves [1], chloroplasts [3], photosynthetic bacteria [4]. In recent years, the study on DL in green plants has been an active field of biophotonics. It can be used as an important index to evaluate biological activity, and can reflect the internal growth and development information of organisms. Current research results show that the DL is very sensitive and dependent on many environmental and physiological factors [5-8]. W. Wang et al. [9] carried out DL image detection on the leaves of Excoecaria cochinchinnensis leaves and some other plant species. The results show that the DL can offer the important information of photosynthesis, cell division and energy transformation of plant species. H. Wang et al. [10] studied the DL phenomenon of leaves at different leaf positions on the same branch of kangaroo flower, and took leaves of different growth and development stages. They believed that the DL attenuation parameters from new leaves to old leaves increased with the increase in leaf age, then it remained relatively stable for a period of time, and finally began to decline. M. Luo et al. [11] discussed the relationship between the DL of sugarcane leaves and chlorophyll content, the weight of a single stem. The results showed that the DL intensity of sugarcane leaves was related to the metabolism and energy conversion ability of sugarcane growth. The samples with strong photosynthesis and metabolism had the stronger delayed luminescence, and the weight of single 
stem was closely related to the DL. The intensity of DL was closely related to the growth of chloroplast and photosynthesis. However, the content of chlorophyll only indicated the amount of chlorophyll in leaves, which cannot represent the activity of chlorophyll, so it was without the relationship with DL. In conclusion, the biological DL was a chemical and physical process closely related to the physiological state of plants, which was the expression of the overall growth and development characteristics of organisms. Therefore, it can reflect the changes and functions of biological physiological state, and it is a powerful parameter representing the changes in plant system.

In this paper, we measured the DL of the picked and potted Scindapsus leaves in the dark room conditions, and fitted the curve. At the same time, the DL curve of the leaves irradiated by different wavelengths light for a long time was also measured. Through curve fitting, the initial intensity and the decay time of stimulated emission photon were closely related to the physiological state of leaves. This may be used to determine the degree and process of plants wilting.

\section{Experimental system design}

The schematic diagram of the system is shown in Fig. 1. The pulsed laser wavelength of $520 \mathrm{~nm}$ was pumped to the sample through a fiber coupler, and the spot diameter was $6 \mathrm{~mm}$. After one single pulse laser, the photons emitted from the leaves were converged by the field lens. In order to increase the number of collected photons, a liquid optical fiber (radial core: $5 \mathrm{~mm}$ ) which radial core much larger than the optical fiber was used to collect the emission photons. Since the photosensitive area of single photon avalanche diode (SPAD) was only $400 \mu \mathrm{m}$, an inverted microscope with a magnification of 20 times was used to reduce to the light spot to $400 \mu \mathrm{m}$ and introduced into the optical fiber. Finally, the photons were collected by SPAD. The laser and SPAD were controlled by the computer through the circuit controller to realize the time synchronization of trigger and acquisition. SPAD is provided by

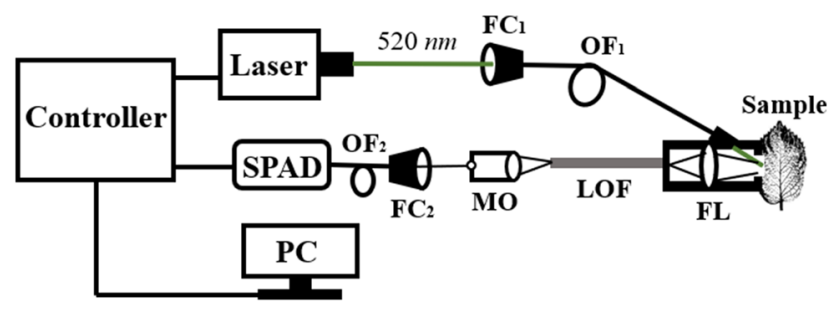

Fig. 1 Schematic of the experimental. The laser wavelength is $520 \mathrm{~nm}, F C_{1}, F C_{2}$ Fiber coupler, $O F_{1}, O F_{2}$ Optical fiber, $F L$ Field lens, $L O F$ Liquid optical fiber, $M O$ Micro-objective, SPAD Single photon avalanche diode
Excelitas Technology (SPCM-AQRH-16X single photon counting module), adopts fiber coupling mode, the effective spectral range from 450 to $900 \mathrm{~nm}$, the dark counts less than 25 cps (counts per second), the accuracy of gate control less than $2 \mu$ s, the quantum efficiency in the 500-900 nm wavelength range higher than 40\%, and the quantum efficiency can reach $70 \%$ at the wavelength of $650 \mathrm{~nm}$. Although the photosensitive diameter is only $150 \mu \mathrm{m}$, but because of a built-in coupling lens, it can image a $400 \mu \mathrm{m}$ diameter spot to the effective detection area of the detector. As shown in Fig. 2, the time synchronization of the laser and SPAD was controlled by the duty cycle of the electric pulse. The excitation time of the pulsed laser was $100 \mathrm{~ms}$, and the pulse width was $10 \mathrm{~ms}$. Because most of the fluorescence lasts a few hundred nanoseconds, in order to shield the effect of fluorescence as much as possible, SPAD starts to collect the excited emission photon with the delay of $150 \mu$ s and the width of the acquisition gate $30 \mathrm{~ms}$ after the end of the pulse laser. Since each laser pulse produced very few stimulated emission photons, and the direction of the emitted photons was also random, the number of photons collected was very limited, resulting in a very low signal-to-noise ratio. Therefore, we counted 100 times in one acquisition gate, and repeated 50 times pulses for accumulation to obtain the change curve of photon number with time delay [12]. Each DL curve was the average of 50 times repeated measurement.

\section{Results and analysis}

\section{The DL characteristic curve}

The leaves of Scindapsus were selected for the experiment sample, and the power of the laser was $43 \mathrm{~mW}$. The ending of a short time after the laser pulse, the strong fluorescence photon will be collected, which included both of the leaf and instrument. The attenuation curve of chlorophyll fluorescence in greenhouse is characterized by complex fitting of multiple indices, and each index has an independent fluorescence lifetime [13]. The singlet annihilation will be created when the pulse laser energy is large, which provides an additional deactivation pathway for the excited states and leads therefore to a shorting of the fluorescence lifetimes and a decrease in the fluorescence yield [14]. These factors will cause the fluorescence intensity to be unstable. In order to reduce the influence of fluorescence, we measured the change in excitation photon intensity with the number of pulses at different acquisition delay times. When the delay time was $50 \mu \mathrm{s}, 100 \mu \mathrm{s}$ and $150 \mu \mathrm{s}$, respectively, 50 times were repeatedly measured in each delay time, and the maximum numbers of photons with the 


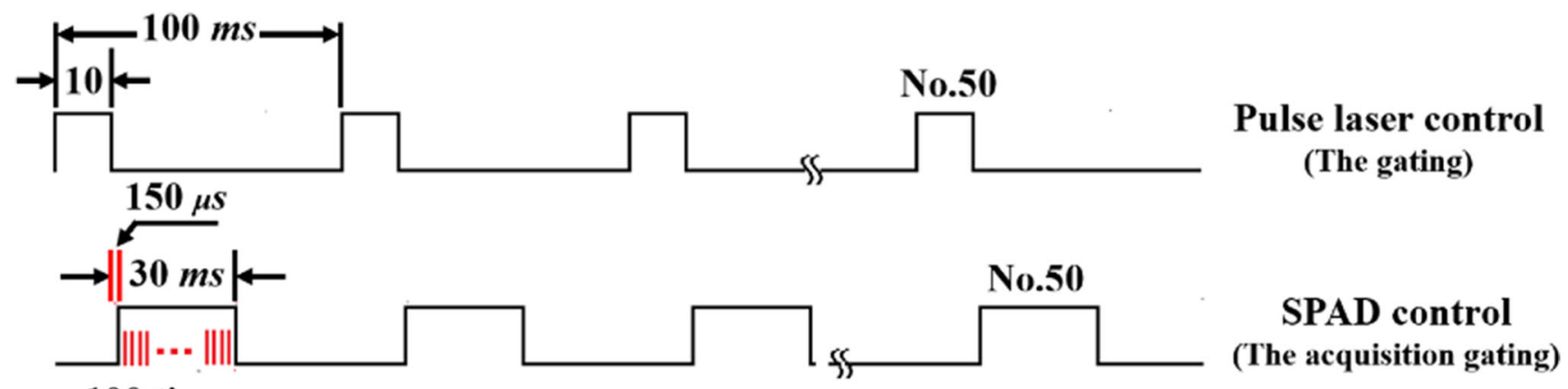

100 times

\section{$\bullet \bullet$ No.2, No.3,...No.50 acquisition gatings}

Fig. 2 The time synchronization of laser pulse and SPAD was controlled by electric pulse

delay time were shown in Fig. 3. It can be seen that, when the acquisition delay was $150 \mu \mathrm{s}$, the photon number of the leaf was stable. In the experiments, the delay time was selected as $150 \mu \mathrm{s}$. At the same time, the photon number of the background was measured about $700 \mathrm{cps}$, and the effect of the background noise was negligible compared with the photon number of the sample.

\section{The DL curve in darkroom conditions}

The experiment was carried out in a dark room (room temperature $22{ }^{\circ} \mathrm{C}$ ). In Fig. 4(a), the black line shows the DL curve (all data are the average of 50 repeated measurements) when the leaves of Scindapsus have just been picked. The hyperbolic and exponential decay functions were used to, respectively fitted, the red dot line was the fitted result of hyperbolic function, and the blue dot line was the exponential function. In order to compare these two different fitted results, the horizontal axis was taken in

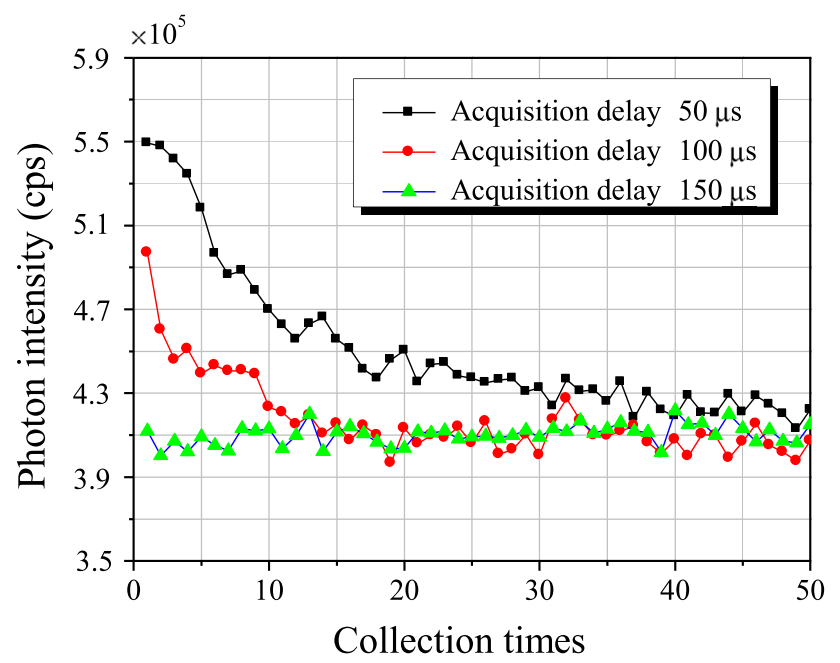

Fig. 3 The curve of maximum photons with the times of measurements in different delay times logarithmic form, as shown in Fig. 4(b). By calculation, the fitted degree of hyperbolic decay function was higher than that of exponential decay function, and the goodness reached to $R^{2}=0.99977$.

The hyperbolic function form is as follows [15]:

$I(t)=\frac{I(0)}{\left(1+\frac{t}{\tau}\right)^{\beta}}$

where, $I(0)$ and $I(t)$ represent the intensity of stimulated emission photon at the initial moment and the moment $t$, respectively. The parameter $\tau$ is a characteristic time, which is related to the property of the sample itself, and $\beta$ is an exponential factor, both of them can be obtained by fitting. The initial intensity $I(0)$ is determined by factors such as the composition of sample, intensity of pulsed laser, pulse width of laser, and the delay time of measurement. The DL curve was used to quantitatively describe the properties of sample, and could be provided us the information which reflected the intrinsic nature of biological system [16].

The experiment measured the DL curves of the leaves were just picked, placed in the dark room for 2, 4, and $6 \mathrm{~h}$, respectively. At the same time, the DL curve of the leaves on plants was measured in the same measuring time period, and the curves were fitted by hyperbolic decay function. The fitting results of initial intensity $I(0)$ were, respectively, shown in Fig. 5(a) and (b). Every number was the average of 50 times repeated measurements. It is shown in Fig. 5(a), with the increase in the measurement time, the initial intensity $I(0)$ of collecting emitted photons for the picked leaves was gradually decreases, while the $I(0)$ value of the potted leaves was basically constant.

In the darkroom, leaves no longer carry out photosynthesis, but respiration. The respiration is to completely or partially decompose the organic matter, and the energy generated provides the ability of life activities. For the picked leaves, the organic matter is consumed with time, 

hyperbolic and exponential function fitting results when the leaves have just been picked; b Carry out logarithmic comparison
Fig. 4 a The DL curve,
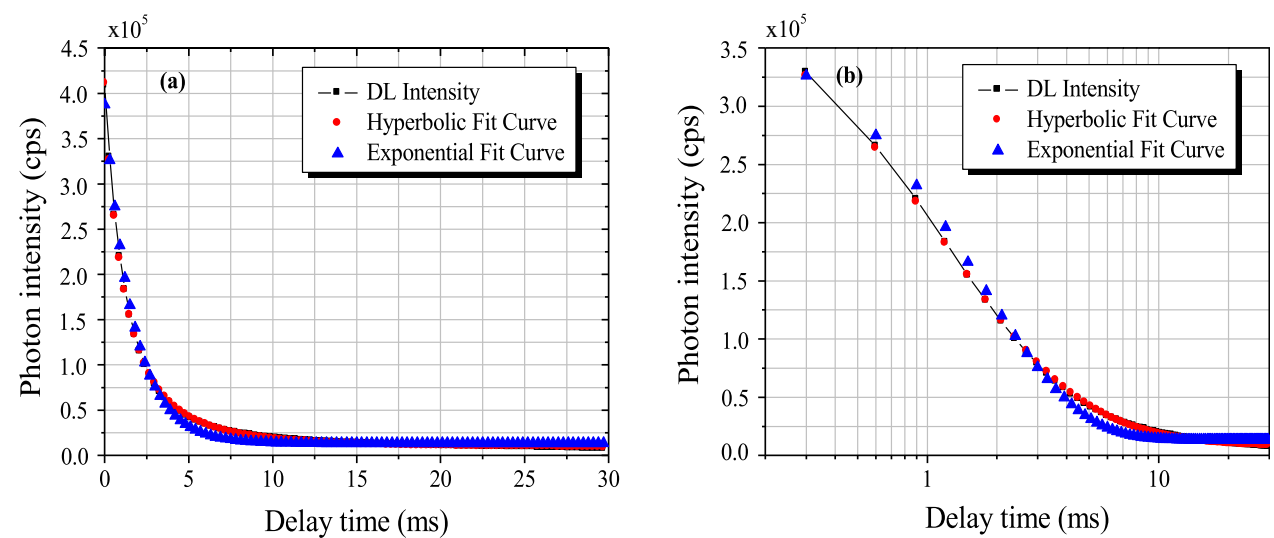

Fig. 5 a The change in the initial strength $I(0)$ of the picked leaves with the measurement time; b The change in the initial strength $I(0)$ of the potted leaves with the measurement time
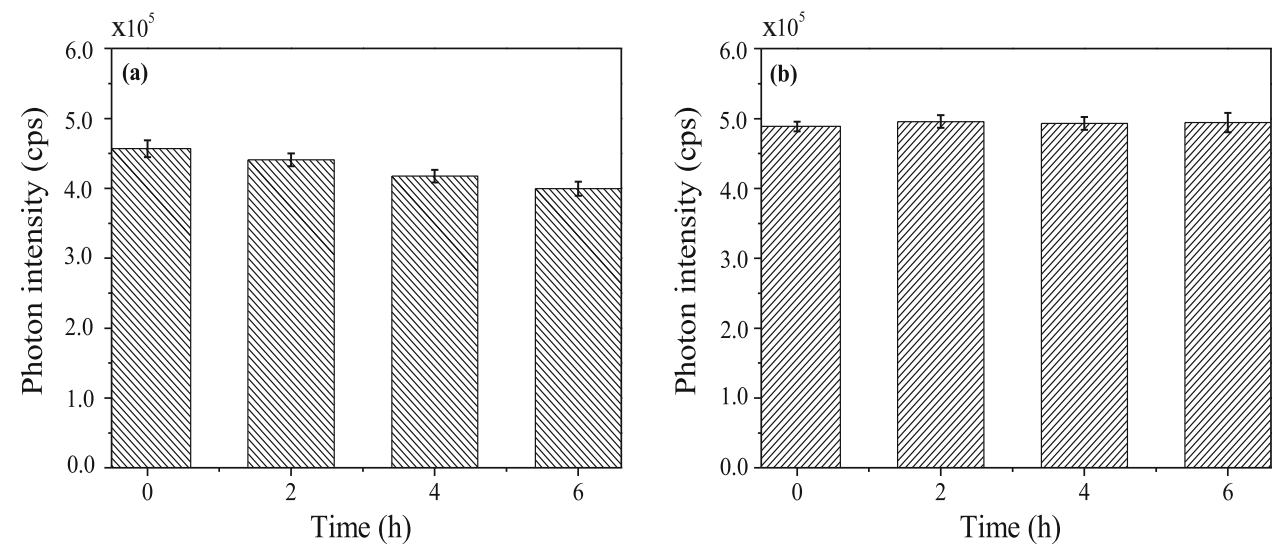

and the metabolites are accumulated, which will wither after a period of time. Different from the picked leaves, the leaves on the plant are not an isolated system. In the process of respiration, the consumed organic matter and metabolites are transported through the plant, the whole life activity of the plant will maintain the higher "activity" of the leaves. Therefore, it is measured that the intensity of the emitted photons of the picked leaves decreases with the measurement time, that is, it slowly moves toward a "death" trend. It turned out that the oscillations are solutions of a Hamiltonian that keeps coherent states coherent, the oscillations disappear as soon as the biological system loses its integrity or its collective structure [5].

The decay time $\tau$ from DL curve fitting of picked and potted leaves in different delay times were shown in Fig. 6. The fitting parameters of potted leaves basically remain unchanged, and the picked leaves decreased with measurement time. The decay parameter $\tau$ represents the time required for the number of photons to decay from maximum to minimum (until reaches the background signal) in the DL curve. The atoms or molecules in the leaves are excited by the energy of pulsed laser, and the excited photons are emitted in the process of de-excitation. The metastable state determines the relaxation time of the deexcitation, that is, the decay time of the emitted photons,

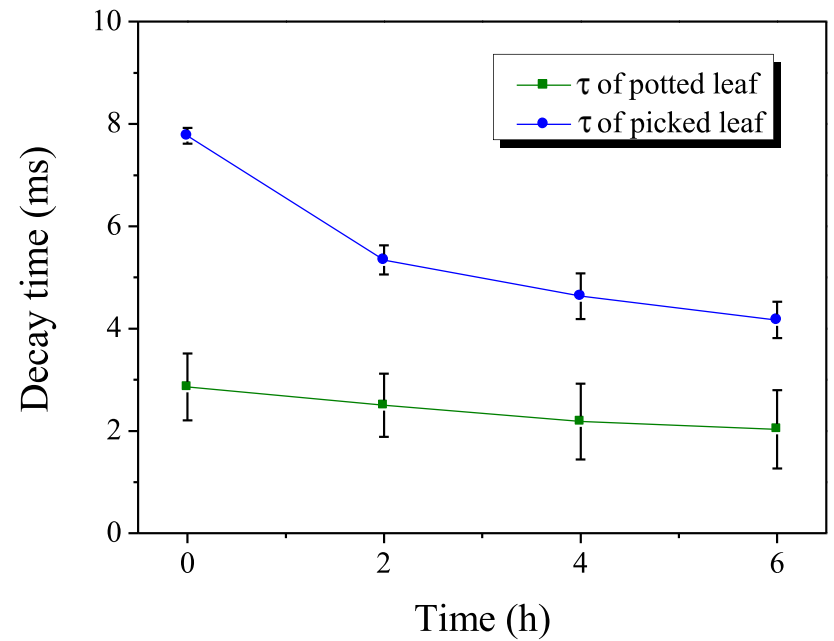

Fig. 6 The change of the decay time $\tau$ of the picked and potted leaves with the measured time

which should be closely related to the chemical reaction process in the leaves. We infer that the exchange of energy and material between the leaves and the plant is relatively stable, so the value of $\tau$ remains basically unchanged. When the leaves are picked, the more metastable states may be produced by the metabolites remaining of the leaves, which take longer for the photons to decay. 
Table 1 The fitted parameter of DL curve

\begin{tabular}{|c|c|c|c|c|c|c|}
\hline \multirow{2}{*}{$\begin{array}{l}\text { Plant state } \\
\text { Parameters }\end{array}$} & \multicolumn{3}{|l|}{ Picked leaves } & \multicolumn{3}{|l|}{ Potted leaves } \\
\hline & $I(0)\left(\times 10^{5} \mathrm{cps}\right)$ & $\tau(\mathrm{ms})$ & $R^{2}$ & $I(0)\left(\times 10^{5} \mathrm{cps}\right)$ & $\tau(\mathrm{ms})$ & $R^{2}$ \\
\hline $0 \mathrm{~h}$ & 4.56 & 7.73 & 0.9998 & 4.88 & 2.86 & 0.9999 \\
\hline $2 \mathrm{~h}$ & 4.41 & 5.31 & 0.9997 & 4.95 & 2.50 & 0.9998 \\
\hline $4 \mathrm{~h}$ & 4.17 & 4.62 & 0.9995 & 4.93 & 2.18 & 0.9998 \\
\hline $6 \mathrm{~h}$ & 3.99 & 4.15 & 0.9996 & 4.94 & 2.03 & 0.9998 \\
\hline
\end{tabular}

Fig. 7 a Using $520 \mathrm{~nm}$ wavelength laser, the DL curves of picked leaves in the condition of before laser irradiation, continuous irradiation $2 \mathrm{~h}, 4 \mathrm{~h}$ and restoration of darkroom; b Using $400 \mathrm{~nm}$ wavelength laser, the DL curves of picked leaves in the condition of before laser irradiation, continuous irradiation $2 \mathrm{~h}, 4 \mathrm{~h}$ and restoration of darkroom

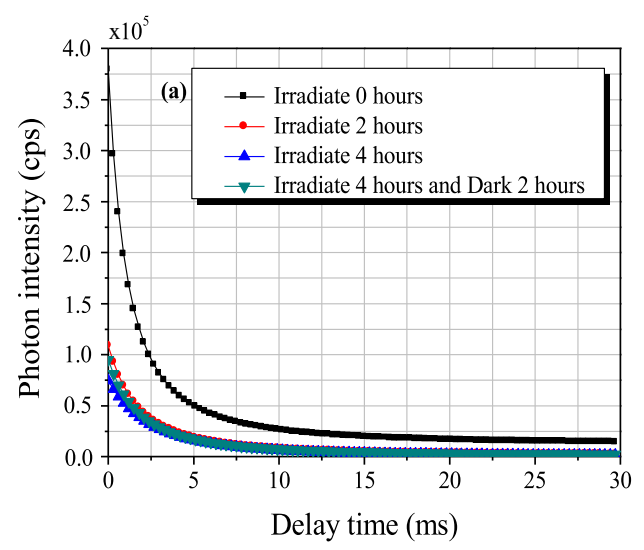

The fitted parameters of the DL curve are listed in Table 1, and every value is the average of 50 times measurements.

\section{The DL curve in laser irradiation conditions}

In the condition of darkroom, we used a laser wavelength of $520 \mathrm{~nm}$ to continuously irradiate the picked leaves. The irradiated area covered the whole detection area with a diameter of $6 \mathrm{~mm}$. The DL curve was measured before the laser irradiation, continuous irradiation for $2 \mathrm{~h}$ and $4 \mathrm{~h}$. After $4 \mathrm{~h}$, the leaves were repeatedly exposed to darkroom conditions for $2 \mathrm{~h}$, and the DL curve was measured again. The measurement results are as follows as shown in Fig. 7(a). Then we changed the wavelength of the laser, and the above experiment was repeated with a $400 \mathrm{~nm}$ wavelength laser. The measured curve was shown in Fig. 7(b).

It was found that, in the conditions of laser irradiation with two different wavelengths, as the placement time increased, the maximum photon intensity $I(0)$ of the DL curve decreased sharply after $2 \mathrm{~h}$ of illumination, and the downward trend slowed down after $4 \mathrm{~h}$ of illumination. Returned to darkroom for $2 \mathrm{~h}$, the maximum photon intensity showed an upward trend. After the DL curves were fitted by hyperbolic function, the decay time $\tau$ was shown in Fig. 8. The fitted parameters were shown in Table 2.

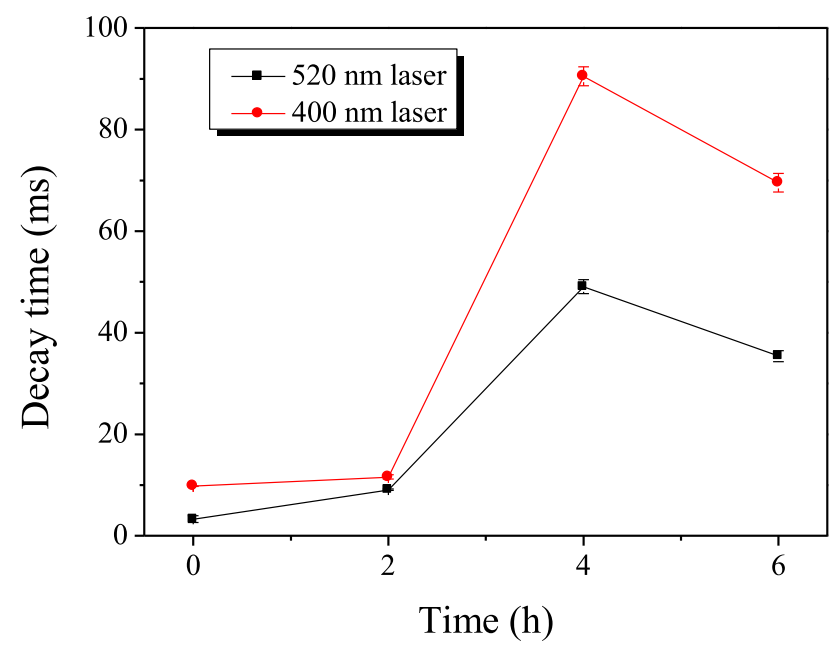

Fig. 8 The picked leaves decay time of DL curves continuously irradiated by $520 \mathrm{~nm}$ and $400 \mathrm{~nm}$ wavelength laser

The experimental and fitting results show that, under the same laser irradiation power, the intensity of the excited photon on the leaves of $400 \mathrm{~nm}$ laser irradiation is slightly higher than that of the $520 \mathrm{~nm}$ laser irradiation. Compared with the leaves that are always in a darkroom condition, the stimulated emission photon is greatly attenuated after the leaves were irradiated by the laser of the specified wavelength. In the condition of darkroom, the leaves mainly 
Table 2 The picked leaves hyperbolic fitted parameters of DL curve by $520 \mathrm{~nm}$ and $400 \mathrm{~nm}$ wavelength laser

\begin{tabular}{llllllll}
\hline Laser & \multicolumn{2}{l}{$520 \mathrm{~nm}$} & & \multicolumn{2}{l}{$400 \mathrm{~nm}$} \\
\cline { 2 - 3 } Parameters & $I(0)\left(\times 10^{5} \mathrm{cps}\right)$ & $\tau(\mathrm{ms})$ & $R^{2}$ & & $I(0)\left(\times 10^{5} \mathrm{cps}\right)$ & $\tau(\mathrm{ms})$ & $R^{2}$ \\
\hline Irradiate 0 h & 3.79 & 3.28 & 0.9990 & & 6.15 & 9.75 & 0.9998 \\
Irradiate 2 h & 1.09 & 9.07 & 0.9989 & & 1.18 & 11.60 & 0.9989 \\
Irradiate 4 h & 0.75 & 49.05 & 0.9989 & & 0.83 & 90.49 & 0.9999 \\
Irradiate 4 h & 0.95 & 35.38 & 0.9993 & & 0.85 & 69.53 & 0.9999 \\
\hline
\end{tabular}

carry out respiration, and absorb $\mathrm{O}_{2}$ to produce $\mathrm{CO}_{2}$. Different from darkroom conditions, in the environment of continuous laser irradiation, the leaves mainly carry out photosynthesis, the leaves absorb laser energy, $\mathrm{CO}_{2}$ and $\mathrm{H}_{2} \mathrm{O}$ to produce organic matter and release $\mathrm{O}_{2}$ [17]. This may be the main reason for the decay of stimulated emission photons. During the $4 \mathrm{~h}$ of continuous irradiation, the number of emitted photons was stable. When the irradiation ended, the leaves resumed respiration, and the reverse metabolites made the number of photons increase. In addition, the decay time $\tau$ of the DL curve may reflect the response of the pigment in the leaves with the laser wavelength. The wavelength from 400 to $520 \mathrm{~nm}$ has the greatest effect on photosynthesis of plants, and the absorption ratio of chlorophyll and carotenoids was the largest. In the wavelength range of $400 \mathrm{~nm}-450 \mathrm{~nm}$, chlorophyll absorbs the most light energy and hardly does at $520 \mathrm{~nm} \mathrm{[18].} \mathrm{While} \mathrm{at} \mathrm{the} \mathrm{wavelength} \mathrm{of} 520 \mathrm{~nm}$, carotenoid absorbs the most light energy [19]. Therefore, different spectral absorptions result in the different decay time $\tau$ as shown in Fig. 8. After $4 \mathrm{~h}$ of laser irradiation, the decay time decreased after the darkroom condition was restored.

\section{Conclusions}

The results of experiment show that the DL curves of Scindapsus leaves follow the law of hyperbolic function attenuation. In the darkroom environment, for picked leaves, the initial intensity $I(0)$ and decay time $\tau$ of the DL curves gradually decrease, while the potted leaves remain almost unchanged. In the action of respiration, the picked leaves only consume energy but do not produce energy. As time increases, the leaves gradually lose freshness and become wilted. The potted leaves will maintain the freshness for a period of time due to the overall life activities of the plant and maintain normal survival. For continuously irradiated by the laser of different wavelengths, the initial intensity $I(0)$ of the DL curves of picked leaves at $520 \mathrm{~nm}$ and $400 \mathrm{~nm}$ wavelengths decreased sharply after $2 \mathrm{~h}$ of illumination, and after $4 \mathrm{~h}$ of illumination, the downward trend slowed down. And the maximum photon intensity showed an upward trend after $2 \mathrm{~h}$ of darkroom restoration.
We speculate that the unnatural light may destroy the tissue structure of the leaves, inhibit the active response, and make the re-emission ability of biomolecules less affected, resulting in a sharp drop in the number of photons in the leaves. When the end of the light, the leaves resume respiration in the dark room, and the reverse metabolites made the number of photons rise back. The decay time $\tau$ value shows a trend of increasing at first and then decreasing. The difference light wavelengths are mainly related to the response of the wavelength of induced laser to various pigments in the leaves. The Biological DL is an inherent characteristic of organisms, which is closely related to many basic biochemical processes such as metabolism of biological systems, cell division and death, photosynthesis. The measurement and analysis of DL can provide a deep understanding of these biological processes, which may provide a useful basis for applications in the fields of biology and agronomy.

Funding ENN Research Fund (EIST202008).

\section{Declarations}

Conflict of interest The authors declare no conflicts of interest.

Open Access This article is licensed under a Creative Commons Attribution 4.0 International License, which permits use, sharing, adaptation, distribution and reproduction in any medium or format, as long as you give appropriate credit to the original author(s) and the source, provide a link to the Creative Commons licence, and indicate if changes were made. The images or other third party material in this article are included in the article's Creative Commons licence, unless indicated otherwise in a credit line to the material. If material is not included in the article's Creative Commons licence and your intended use is not permitted by statutory regulation or exceeds the permitted use, you will need to obtain permission directly from the copyright holder. To view a copy of this licence, visit http://creativecommons. org/licenses/by/4.0/.

\section{References}

1. B.L. Strehler, W.J. Arnold, Light production by green plants. J. Gen. Physiol. 34(6), 809-820 (1951)

2. J.F. Allen, J. Bennett, K.E. Steinback, C.J. Arntzen, Chloroplast protein phosphorylation couples plastoquinone redox state to distribution of excitation energy between photosystems. Nature 291(5810), 25-29 (1981) 
3. B.L. Strehler, The luminescence of isolated chloroplasts. Arch. Biochem. Biophys. 34(2), 239-248 (1951)

4. W. Arnold, J. Thompson, Delayed light production by blue-green algae, red algae, and purple bacteria. J. Gen. Physiol. 39(3), 311-318 (1956)

5. Y. Yan, F.A. Popp, S. Sigrist, D. Schlesinger, A. Dolf, Z. Yan, S. Cohen, A. Chotia, Further analysis of delayed luminescence of plants. J. Photochem. Photobiol., B 78(3), 235-244 (2005)

6. F.A. Popp, K.H. Li, Q. Gu, Recent Advances in Biophoton Research and Its Applications (World Scientific, London, 1992)

7. A. Khetani, V.S. Tiwari, A. Harb, H. Anis, Monitoring of heparin concentration in serum by Raman spectroscopy within hollow core photonic crystal fiber. Opt. Express. 19(16), 15244-15254 (2011)

8. D.H. Qi, A.J. Berger, Chemical concentration measurement in blood serum and urine samples using liquidcore optical fiber Raman spectroscopy. Appl. Opt. 46(10), 1726-1734 (2007)

9. W. Wang, D. Xing, S. Tan, J. Han, Research on delayed luminescence of excoecaria cochinchinnensis leaves. J. Opt. 17(4), 310-314 (2000)

10. H. Wang, Experimental study on delayed luminescence of different leafposition from plant leaves. Exp. Technol. Manag. (2012)

11. M. Luo, D. Li, J. Liang, Z. Tan, P. Chen, R. Duan, C. Liu, Study on the relationship between UBE of sugarcane leaves and content of chlorophyll and single-stem weight. Acta Laser Biol. Sinica. 11(003), 173-176 (2002)

12. X. Zhang, P. Zheng, Y. Yang, J. Wang, M. Zhao, F. Gu, P. Gao, M. Jiang, Y. Liu, B.Q. Tang, Quantum correlating measurements of human-body delayed Lu-minescence and preliminary experimental results. OSA Continuum (2020)

13. G. Schmuck, J. Verdebout, Laser-induced time-resolved fluorescence of vegetation. IEEE Trans. Geosci. Remote Sens. 29(4), 674-678 (1991)

14. F. Pellegrino, Energy Transfer in the Primary Stages of the Photosynthetic Process Investigated by Picosecond Time Resolved Fluorescence Spectroscopy (Ph.D. Thesis City University of New York, 1981)

15. P. Chen, L. Zhang, F. Zhang, J. Liu, H. Bai, G. Tang, L. Lin, Spectral discrimination between normal and leukemic human sera using delayed luminescence. Biomed. Optics Expres. 3(8), 1787-1792 (2012)

16. F.A. Popp, Y. Yan, Delayed luminescence of biological systems in terms of coherent states. Phys. Lett. A 293(1-2), 93-97 (2002)

17. N.K. Boardman, Comparative photosynthesis of sun and shade plants. Ann. Rev. Plant Physiol 28(1), 355-377 (1977)

18. L.D. Boni, D.S. Correa, F.J. Pavinatto Boni, D.S.D. Santos, C.R Mendonça, Excited state absorption spectrum of chlorophyll a obtained with white-light continuum. J. Chem. Phys. 126(16), 165102 (2007)

19. G. Araki, T. Murai, Molecular structure and absorption spectra of carotenoids. Progress Theoret. Phys. 10(6), 639-654 (1952)

Publisher's Note Springer Nature remains neutral with regard to jurisdictional claims in published maps and institutional affiliations. 